\title{
ARC FAULT TOLERANCE OF CARTESIAN PRODUCT OF REGULAR DIGRAPHS ON SUPER-RESTRICTED ARC-CONNECTIVITY
}

\author{
GuOzhen Zhang And ShIYing Wang \\ School of Mathematical Sciences \\ Shanxi University \\ Taiyuan, Shanxi 030006, P.R. China \\ e-mail: guozhen@sxu.edu.cn
}

\begin{abstract}
Let $D=(V(D), A(D))$ be a strongly connected digraph. An arc set $S \subseteq A(D)$ is a restricted arc-cut of $D$ if $D-S$ has a non-trivial strong component $D_{1}$ such that $D-V\left(D_{1}\right)$ contains an arc. The restricted arcconnectivity $\lambda^{\prime}(D)$ is the minimum cardinality over all restricted arc-cuts of D. In [C. Balbuena, P. García-Vázquez, A. Hansberg and L.P. Montejano, On the super-restricted arc-connectivity of s-geodetic digraphs, Networks $\mathbf{6 1}$ (2013) 20-28], Balbuena et al. introduced the concept of super- $\lambda^{\prime}$ digraphs. In this paper, we first introduce the concept of the arc fault tolerance of a digraph $D$ on the super- $\lambda^{\prime}$ property. We define a super- $\lambda^{\prime}$ digraph $D$ to be $m$-super- $\lambda^{\prime}$ if $D-S$ is still super- $\lambda^{\prime}$ for any $S \subseteq A(D)$ with $|S| \leq m$. The maximum value of such $m$, denoted by $S_{\lambda^{\prime}}(D)$, is said to be the arc fault tolerance of $D$ on the super- $\lambda^{\prime}$ property. $S_{\lambda^{\prime}}(D)$ is an index to measure the reliability of networks. Next we provide a necessary and sufficient condition for the Cartesian product of regular digraphs to be super- $\lambda^{\prime}$. Finally, we give the lower and upper bounds on $S_{\lambda^{\prime}}(D)$ for the Cartesian product $D$ of regular digraphs and give an example to show that the lower and upper bounds are best possible. In particular, the exact value of $S_{\lambda^{\prime}}(D)$ is obtained in special cases.
\end{abstract}

Keywords: fault tolerance, restricted arc-connectivity, super-restricted arcconnectivity, Cartesian product, regular digraph.

2010 Mathematics Subject Classification: 05C40, 68M15.

\section{REFERENCES}

[1] D. Bauer, F. Boesch, C. Suffel and R. Tindell, Combinatorial optimization problems in the analysis and design of probabilistic networks, Networks 15 (1985) 257-271. doi:10.1002/net.3230150210 
[2] J. Bang-Jensen and G. Gutin, Digraphs: Theory, Algorithms and Applications (Springer, London, 2000).

[3] C. Balbuena, P. García-Vázquez, A. Hansberg and L.P. Montejano, On the superrestricted arc-connectivity of s-geodetic digraphs, Networks 61 (2013) 20-28. doi:10.1002/net. 21462

[4] C.W. Cheng and S.Y. Hsieh, Bounds for the super extra edge connectivity of graphs, in: International Computing and Combinatorics Conference, D. Xu, D. Du, D. Du $(\mathrm{Ed}(\mathrm{s}))$, Lecture Notes in Comput. Sci. 9198 (2015) 624-631. doi:10.1007/978-3-319-21398-9_49

[5] X. Chen, J. Liu and J. Meng, The restricted arc connectivity of Cartesian product digraphs, Inform. Process. Lett. 109 (2009) 1202-1205. doi:10.1016/j.ipl.2009.08.005

[6] A.H. Esfahanian and S.L. Hakimi, On computing a conditional edge-connectivity of a graph, Inform. Process. Lett. 27 (1988) 195-199. doi:10.1016/0020-0190(88)90025-7

[7] Y. Hong, J. Meng and Z. Zhang, Edge fault tolerance of graphs with respect to super edge connectivity, Discrete Appl. Math. 160 (2012) 579-587. doi:10.1016/j.dam.2011.10.033

[8] Z.M. Hong and J.M. Xu, Vulnerability of super edge-connected networks, Theoret. Comput. Sci. 520 (2014) 75-86. doi:10.1016/j.tcs.2013.10.021

[9] J. Liu and J. Meng, Super-connected and super-arc-connected Cartesian product of digraphs, Inform. Process. Lett. 108 (2008) 90-93. doi:10.1016/j.ipl.2008.04.006

[10] J. Liu, J. Meng and Z. Zhang, Double-super-connected digraphs, Discrete Appl. Math. 158 (2010) 1012-1016. doi:10.1016/j.dam.2010.02.004

[11] L. Volkmann, Restricted arc-connectivity of digraphs, Inform. Process. Lett. 103 (2007) 234-239. doi:10.1016/j.ipl.2007.04.004

[12] S. Wang and S. Lin, $\lambda^{\prime}$-optimal digraphs, Inform. Process. Lett. 108 (2008) 386-389. doi:10.1016/j.ipl.2008.07.008

[13] J.M. Xu, Connectivity of Cartesian product digraphs and fault-tolerant routings of generalized hypercubes, Appl. Math. J. Chinese Univ. 13 (1998) 179-187. doi:10.1007/s11766-998-0039-x

[14] J.M. Xu, Topological Structure and Analysis of Interconnection Networks (Kluwer Academic Publishers, Dordrecht, 2001). doi:10.1007/978-1-4757-3387-7

[15] C. Yang and J.M. Xu, Reliability of interconnection networks modeled by Cartesian product digraphs, Networks 52 (2008) 202-205. doi:10.1002/net.20231 
[16] G. Zhang, Arc fault tolerance of Cartesian product digraphs on hyper arc connectivity, Int. J. Comput. Math. 91 (2014) 2152-2162. doi:10.1080/00207160.2014.883069

[17] Z. Zhang and Y. Zhu, Cyclic arc-connectivity in a Cartesian product digraph, Appl. Math. Lett. 23 (2010) 796-800.

doi:10.1016/j.aml.2010.03.013

Received 10 October 2016

Accepted 7 June 2017 